Recepción: 10 / 04 / 2018

Aceptación: 07 / 05 / 2018

Publicación: 02 / 07 / 2018

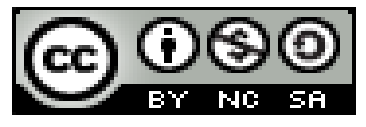

Ciencias económicas y empresariales

Artículo de Investigación

\title{
Valorando las competencias del Talento Humano
}

Valuing the skills of Human Talent

\section{Valorizando as habilidades do talento humano}

\author{
Marcos B. Vera-Mendoza ${ }^{\mathrm{I}}$ \\ mveram88@hotmail.com \\ Franklin G. Jiménez-León II \\ franklin.jimenez@ec.mcd.com
}

Correspondencia:mveram88@hotmail.com

\begin{abstract}
${ }^{\text {I}}$ Máster Universitario en Formulación y Tecnología del Producto, Ingeniero Industrial, Docente de la
Universidad Laica Eloy Alfaro de Manabí, Manta, Ecuador.

II Magister en Administración de Empresas con Mención en Recursos Humanos, Ingeniero Comercial, Docente de la Universidad de Guayaquil, Guayaquil, Ecuador.
\end{abstract}




\title{
Resumen
}

El presente trabajo de investigación, de carácter descriptivo y documental pretendió poner al día el tema de la valoración de las competencias del talento humano. Para ello se exploró las diferentes miradas que ha tenido la evaluación del desempeño desde las primeras propuestas académicas realizadas en los años sesenta del siglo XX en Estados Unidos, como planteamiento de superación de la mirada de la educación y formación del talento y capital humano como un gasto y consumo para asumirlo como una inversión en el mejoramiento de las capacidades laborales. Siendo que en tanto técnica evaluativa ha cambiado en sus fines iniciales vinculados con las mejoras salariales y la promoción del personal hacia otros que pretenden contribuir a la motivación del trabajador a la mejora en su puesto de trabajo. También ha evolucionado en tanto técnica desde la evaluación unilateral hacia el análisis de $360^{\circ}$. Se explora aspectos colaterales como la auditoría de desempeño y la contribución de la valoración del desempeño del talento y capital humano para el desarrollo económico.

Palabras clave: desempeño; evaluación; competencias; talento humano; capital humano.

\begin{abstract}
The present research work, of a descriptive and documentary nature, sought to update the subject of the assessment of human talent competencies. To this end, the different perspectives that the evaluation of performance has had since the first academic proposals made in the 60s of the 20th century in the United States were explored, as an approach to overcome the view of education and training of talent and human capital as a spending and consumption to assume it as an investment in the improvement of labor skills. Being that, as an evaluative technique, it has changed in its initial aims linked to salary improvements and the promotion of personnel towards others who intend to contribute to the worker's motivation to improve their job. It has also evolved as a technique from the unilateral evaluation to the $360^{\circ}$ analysis. It explores collateral aspects such as the performance audit and the contribution of the assessment of the performance of talent and human capital for economic development.
\end{abstract}

Keywords: performance; evaluation; competencies; human talent; human capital. 


\section{Resumo}

O presente trabalho de pesquisa, de natureza descritiva e documental, buscou atualizar o tema da avaliação de competências de talentos humanos. Para tanto, foram exploradas as diferentes perspectivas que a avaliação de desempenho teve desde as primeiras propostas acadêmicas feitas nos anos 60 do século XX nos Estados Unidos, como uma abordagem para superar a visão de educação e formação de talento e capital humano como gastos e consumo para assumi-lo como um investimento na melhoria das habilidades de trabalho. Sendo que, como técnica avaliativa, mudou em seus objetivos iniciais ligados à melhoria salarial e à promoção de pessoal para outros que pretendem contribuir para a motivação do trabalhador para melhorar seu trabalho. Também evoluiu como técnica desde a avaliação unilateral até a análise $360^{\circ}$. Explora aspectos colaterais como a auditoria de desempenho e a contribuição da avaliação do desempenho de talentos e capital humano para o desenvolvimento econômico.

Palavras chave: performance; avaliação; competências; talento humano; capital humano.

\section{Introducción}

Existe un consenso entre los estudiosos de las organizaciones empresariales acerca de la enorme importancia que tiene la valoración adecuada de las capacidades y competencias del talento humano, también conocido como Capital Humano.

Si bien en la visión de los economistas y contadores tradicionales se catalogaba los recursos asignados a la capacitación y formación de los colaboradores como un gasto o consumo, es a partir de los años 60 del siglo XX cuando destacados economistas promueven el reconocimiento del carácter de inversión de los referidos recursos.

Así mismo, en correspondencia con el desarrollo del enfoque de talento humano y capital humano fueron diseñando técnicas de evaluación y luego de análisis del desempeño como dispositivo que le permite a los gerentes valorar las capacidades y competencias, así como las debilidades, fortalezas, potencialidades de las personas que tienen el rol de colaboradores. Tales evaluaciones y análisis han sido utilizadas como mecanismo de premio y castigo, pero también como instrumento para la superación de los aspectos negativos u oportunidades de mejora, logrando con ello elevar los niveles de excelencia de las organizaciones en las que se aplica adecuadamente. 


\section{Aspectos conceptuales}

En este artículo se pretende analizar las ventajas de valorar las competencias del talento humano desde las perspectivas de la evaluación de desempeño porque de esta manera se ofrece una buena oportunidad para revisar los planes de carrera de las personas y verificar sus potencialidades, habilidades y debilidades.

Desde este punto de vista debemos ver la importancia de la evaluación de desempeño como una técnica que suministra información y da las bases para la toma de decisiones, y adicionalmente puedan revisar el comportamiento que tiene el personal con su trabajo. Y los resultados permitan realizar retroalimentaciones con respeto al desempeño para mejorar el rendimiento. Por otra parte, la evaluación de desempeño juega un papel en cuanto mejorar la motivación en el trabajo porque la mayoría de las veces las personas desean retroalimentación con respeto a la manera cómo actúan. (Dessler, 1994)

En ese mismo orden de ideas, Grote (2002) nos platea que para toda empresa que sea bien organizada, un sistema de evaluación del desempeño es la herramienta administrativa más relevante e importante de todas, porque con este proceso se podrá tener una influencia muy significativa sobre la carrera de los individuos y sobre su vida laboral. Esto contribuye con el propósito que dicho sistema ofrezca a las empresas que los trabajadores se identifiquen plenamente con la misión, visión y valores de la Institución a la que pertenecen.

Por otra parte, Grote (2002) considera que la evaluación de desempeño bien diseñada debería partir de una serie de competencias identificadas, con la expectativa de que la totalidad de sus miembros las posean y demuestren a través de su desempeño. Mientras que para Desser (1994), el llevar a cabo este proceso permite diagnosticar los estilos de gerencia, identificar los problemas potenciales de las personas, determinar las acciones correctivas que sean necesarias, establecer un plan de acción para crear un ambiente de trabajo seguro, y que los pagos salariales sean justos para el tipo de cargo que desempeñe un trabajador.

En igual sentido, Robbins (1995) indica que una de las metas principales de la evaluación del desempeño es determinar con precisión la contribución del desempeño individual como base para tomar decisiones de asignación de recompensas. Además, la evaluación del desempeño supone una herramienta al servicio de la persona, con una concepción más responsable de su trabajo y de 
la empresa, para la optimización de recursos. Es también una técnica para apreciar sistemáticamente, dentro de la mayor objetividad posible, la actuación de una persona durante un determinado tiempo, en relación con su trabajo actual y sus características personales, así como su contribución a objetivos previstos.

Al profundizar en el tema de la evaluación de competencias, encontramos que esta se enmarca en la evaluación del desempeño, por lo tanto, supone una comparación entre las competencias demostradas por el individuo en su trabajo y las competencias exigidas para un desempeño exitoso en el puesto que ocupa.

No obstante, para algunos autores tiende a abandonarse la noción misma de evaluación, dedo que ello implica "enjuiciar, criticar, y casi siempre centrarse en lo negativo" (Muñoz, 2004, p. 44). En tal sentido propone que sea sustituido por la categoría analizar, la cual implicaría tomar en cuenta tanto lo positivo como lo negativo, las fortalezas y debilidades, siendo un enfoque que tendería ser más equilibrado. (Muñoz; 2004)

Este mismo autor reseña que los cambios trascienden el enfoque evaluador para afectar también los fines mismos de la valoración del desempeño, cuestionando las tradicionales tendencias a hacerlo vinculante a las mejoras salariales, que tendían a prestarse más a prácticas de premio/castigo que a sistemas de viabilización de oportunidades de mejora. En consecuencia, una mirada adecuada a lo que denomina análisis de desempeño debería propender a mutuos compromisos de la gerencia y el colaborador.

Estos podrían ser, en el caso del empleado evaluado compromisos como estudiar, capacitarse, actualizarse o desarrollar nuevas actitudes, valores, hábitos y actuaciones. Y por parte del jefe y de la organización, podrían ser compromisos relacionados con la asignación de recursos, de oportunidades de entrenamiento $\mathrm{u}$ otros apoyos necesarios para el mejoramiento que se quiere lograr en el desempeño del colaborador. (Muñoz, 2004, p. 45)

Otras investigaciones recientes relacionan la evaluación de desempeño con un adecuado clima organizacional. Se reporta la investigación sobre el caso de la empresa PTS Perú para el año 2015, en la cual se habría demostrado que variables del clima organizacional tales como que más relacionadas estuvieron con el desempeño laboral general fueron la calidez, la estructura y el 
apoyo, llegando incluso a afirmar una relación directamente proporcional entre la calidad del clima organizacional y el desempeño laboral. (Montoya, 2016, p. 66)

Otros investigadores (Nolberto, 2017) exploran la relación entre el desempeño laboral y otras variables y dimensiones como la calidad de la gestión del talento humano, la selección de personal, el trabajo en equipo, la capacitación, mediante la aplicación de métodos estadísticos de correlación. Es así como comparte sus hallazgos obtenidos en una organización de la administración pública municipal, obteniendo evidencias de una correlación positiva alta entre una alta calidad de la gestión y un buen desempeño laboral, así como con la capacitación laboral. Mientras que en el caso de la selección de personal la correlación positiva pero más moderada, al igual que con el trabajo en equipo. (Nolberto, 2017, p. 65)

\section{Auditoria del desempeño}

Tomando en cuenta la importancia que tiene la evaluación de desempeño para valorar las competencias del talento humano, requiere de una auditoria que permita contemplar con objetividad cuáles serán los parámetros para auditar al departamento de recursos humanos que se encarga de seleccionar el personal y es quien diseña el instrumento para medir las capacidades, fortalezas y habilidades que ha tenido el personal a través de su formación y preparación desde la educación, conocimiento, ciencia y tecnología.

Se entiende que la evaluación de desempeño debe ser auditada, por lo que Burbano (1995) menciona ciertos aspectos que se deben ser considerados: 
Aspectos principales para auditar

\begin{tabular}{|c|c|}
\hline FUNCIÓN & ASPECTOS PARA EVALUAR \\
\hline Planeación & Presupuestos de personal \\
\hline Reclutamiento & $\begin{array}{l}\text { Fuentes de reclutamiento } \\
\text { Costos de reclutamiento }\end{array}$ \\
\hline Selección & $\begin{array}{l}\text { Entrevistas de empleo } \\
\text { Solicitudes no aceptadas y las causas } \\
\text { Solicitudes de transferencias } \\
\text { Solicitudes de promoción }\end{array}$ \\
\hline Capacitación y desarrollo & $\begin{array}{l}\text { Costo de cursos y programas } \\
\text { Registros de eficiencia en producción } \\
\text { Registros de accidentes } \\
\text { Grado de cumplimiento de los programas } \\
\text { Control de calidad (registro) }\end{array}$ \\
\hline Evaluación del desempeño & $\begin{array}{l}\text { Rendimiento de los departamentos } \\
\text { Comparación de las evaluaciones de los departamentos } \\
\text { Registros de producción } \\
\text { Datos de desperdicio de materiales } \\
\text { Archivo de entrevistas de evaluación }\end{array}$ \\
\hline Compensación & $\begin{array}{l}\text { Archivo sobre salarios y bonificaciones } \\
\text { Resultados de encuestas de salarios } \\
\text { Información sobre horas extras } \\
\text { Información sobre costo de prestaciones social, legales y } \\
\text { extralegales }\end{array}$ \\
\hline Relaciones laborales & $\begin{array}{l}\text { Registro de demandas } \\
\text { Información adicional sobre laudos arbitrales u otros }\end{array}$ \\
\hline Ambientes en el trabajo & $\begin{array}{l}\text { Informes sobre rotación de personal } \\
\text { Informes de ausentismo } \\
\text { Informes de inspecciones de trabajo } \\
\text { Resultados de las encuestas sobre actitudes }\end{array}$ \\
\hline
\end{tabular}

Fuente: Burbano (1995, p.103).

Estos aspectos permitirán a los recursos humanos determinar que van a evaluar en el desempeño del talento humano.

\section{Teorías del capital humano o talento humano}

Para Becker (1964), la teoría de capital humano considera a la educación y las formaciones específicas como una manera que han empleado las personas para incrementar su eficiencia y oportunidad. Esa inversión y tiempo que la gente aplica para adquirir conocimiento, formación e información es lo que le permite dar un mayor rendimiento y productividad en la economía. Las personas sacan provecho de su talento y se convierten en unos agentes económicos porque son capaces de invertir en su educación pensando en su futuro y esperando que se le retribuya en un salario superior. 
En ese mimo orden de ideas, Schultz (1963), para el año 1960 presidente de la Asociación Estadounidense de Economistas, quien en un discurso en dicho cargo afirmó que las inversiones en la formación de los trabajadores, deben constituir un eje central para el desarrollo de un país como altos ingresos. Además, planteó que debería considerarse el consumo como inversión en capital humano o talento humano, mientras criticaba que para la época la formación de talento era contabilizada como gasto, cuando se trataba de inversión en las capacidades productivas de los trabajadores. Así mismo, habría expresado que son los hombres los que adquieren conocimientos y tienen las capacidades y habilidades para mejorar la calidad del trabajo.

En el año siguiente a tal discurso (1961), según aporta Morales (2009), la Oficina Nacional de Investigaciones Económicas organizó una conferencia en Nueva York para tratar sobre la inversión de capital en los seres humanos. Allí se unieron a los planteamientos de Shultz, Gary Becker y George Stigler, quienes habrían argumentado que el capital humano es importante porque la productividad en las economías modernas está basada en la creación, diseminación y utilización del conocimiento. Que el conocimiento es creado en las compañías, en laboratorios y universidades y esparcido en las escuelas y en el trabajo, siendo usado por las compañías para producir bienes y servicios (Becker, 1993)

Desde esta perspectiva el capital humano comprende la integración de políticas, objetivos, normativas, funciones, procedimientos, herramientas y técnicas que en el ámbito laboral se estructuran en función de los procesos de la empresa para elevar a un plano superior la productividad, el desempeño laboral y la eficiencia.

Según estudios actualizados (Vasquez, 2007), poco menos del 90\% de las organizaciones económicas han asumido sistemas de evaluación de desempeño como parte de procesos administrativos o de desarrollo del talento humano. Los primeros refieren principalmente a valoración de condiciones laborales de los empleados o colaboradores, mientras que aquellos que priorizan el desarrollo, la toman como base para optimizar el rendimiento de los colaboradores, así como para reforzar las capacidades técnicas de acuerdo a las exigencias de los puestos de trabajo. 


\section{Economía del conocimiento basada Talento Humano o Capital Humano}

El elemento fundamental para el desarrollo del talento humano o capital humano tiene una interrelación con la educación, el conocimiento, la ciencia y la tecnología. Así, se debe comprender que cuando nos referimos la economía del conocimiento nos referimos a algo que está ligado con el talento humano, es decir, con todo lo que acumula una persona en cuanto a conocimiento, habilidades y capacidades para desarrollarse en su campo laboral y en su vida cotidiana.

Esta producción de conocimiento conllevó a la Organización para la Cooperación y Desarrollo de la Economía desde el año 1965 a proponer mecanismos de evaluación y medición para el capital humano o talento humano, para de esta manera verificar el alcance de la red semántica del talento humano, que sirva de base a su administración, arrojando en sus resultados cual será la inversión en conocimiento. (Madrigal, 2009)

En este sentido Drucker (2006) hace hincapié que la tarea primordial de la gerencia es la de capacitar a las personas para puedan hacer efectivas sus fortalezas e irrelevantes sus debilidades. Y además considera que la gerencia debe estar pendiente de capacitar a su personal y de esta manera tener un trabajador calificado.

Otro de los cambios significativos en los sistemas de valoración de la gestión del talento humano ha sido la evolución de los sistemas fundamentalmente unilaterales en los cuales los jefes inmediatos aplicaban instrumentos estandarizados de evaluación, cuyo resultado sería posteriormente compartido con el colaborador, a sistemas denominados de análisis de desempeño de $360^{\circ}$, los cuales se caracterizan por buscar una mirada de mayor objetividad y abarcante, por lo que incorpora la apreciación de los clientes, compañeros de labores

\section{Conclusiones}

A manera de conclusión, podemos destacar la enorme importancia que las diferentes disciplinas científicas que estudian a las organizaciones le otorgan a la valoración de las competencias y del desempeño de los colaboradores vistos ya no como recurso sino como Talento y Capital Humano. 
Las técnicas e instrumentos de valoración del desempeño han evolucionado desde la noción de evaluación unilateral por parte de los jefes hacia un enfoque de análisis de debilidades y fortalezas con una mirada de $360^{\circ}$, es decir, multiplicando los puntos de referencia de la valoración y cambiando el fin mismo de la aplicación de los instrumentos desde la oferta de mejoras salariales a la de mejora total de los aspectos relacionados con la relación laboral y el desempeño.

La medición del Capital Humano y del Capital Intelectual apoya para la toma de decisiones, para gestionar la inversión en desarrollar habilidades de los empleados, bases de información y capacidades tecnológicas.

Es elemental para medir la competitividad de una empresa y otra. Así mismo es un elemento importante para medir a corto y largo plazo, el rendimiento de la inversión en las personas, sus habilidades y capacidades tecnológicas de la organización.

\section{Referencias Bibliográficas}

Becker, G. (2002). La Inversión en Talento como Valor de Futuro. Capital Humano. Revista para la Integración y Desarrollo de los Recursos Humanos. No. 153. Recuperado de: https://dialnet.unirioja.es/servlet/articulo? codigo $=228582$

Burbano, J. (1995) Auditoria de personal 2da ed) Colombia ECOE Ediciones

Drucker, P. (2006). Drucker para todos los días. Editorial Norma. Bogotá. Colombia.

Grote, D. (2002) The Performance Appraisal Question and Answer Book: a Survival Guide For Managers. New York: American Management Association

Madrigal, B. (2009) Capital humano e intelectual: su evaluación. Observatorio laboral Revista Venezolana. Recuperada de: http://uay.redalyc.org/articulo.oa?id=219016838004

Montoya, D. (2016) Relación entre el clima organizacional y la evaluación del desempeño del personal en una empresa de servicios turísticos: caso PTS Perú 2015”. (Tesis inédita de maestría) Pontificia Universidad Católica del Perú. Lima, Perú. Recuperado en: http://tesis.pucp.edu.pe/repositorio/handle/123456789/7490 
Morales, A (2009) Capital humano, hacia un sistema de gestión en la empresa cubana. Editora Política, la Habana, Cuba

Muñoz, C. (2004) Análisis del desempeño: nuevas tendencias en la llamada evaluación del personal. Pensamiento \& Gestión, $\mathrm{N}^{\circ}$ 16, pp. 43-51. Recuperado en: www.redalyc.org/pdf/646/64601604.pdf

Nolberto, M. (2017) Gestión del talento humano y el desempeño laboral de la municipalidad distrital de Pichanaqui, 2017. (Tesis inédita), Universidad de Huánuco, Perú. Recuperado en: http://repositorio.udh.edu.pe/bitstream/handle/123456789/408/T047_10173016T.pdf?sequence=1 \&isAllowed=y

Robbins, S. (1987). Comportamiento Organizacional conceptos, controversias y aplicaciones. Editorial: PHH Prentice Hall. México

Vásquez, E. (2007) Tendencias en la evaluación del desempeño: un estudio sobre el modo en que las grandes empresas diseñan sus sistemas de evaluación. Capital humano, $\mathrm{N}^{\circ} 212$, recuperada de http://pdfs.wke.es/2/6/8/3/pd0000012683.pdf 